\title{
Der Komplex der nicht-chromatischen Skalen
}

\author{
Kathlén Kohn und Ernst Ulrich Deuker
}

\begin{abstract}
Wir betrachten den Raum aller Skalen (Tonleitern) in der Musik und wollen diesen systematisieren. Dazu verfolgen wir die Idee, gewisse Skalen als Grundgerüst anzusehen und alle übrigen Skalen daraus zu „mischen“.
\end{abstract}

Die musikalische Idee des Grundgerüsts wird im kürzlich erschienenen Buch [1] über Improvisation (nicht nur) im Jazz vorgeschlagen. Aus mathematischer Sicht ist das Grundgerüst der Skalen ein Simplizialkomplex, dessen $\mathrm{Fa}$ cetten die meistverwendeten Skalen in der westlichen $\mathrm{Mu}$ sik - mit Ausnahme des Blues - darstellen. Diesen Zusammenhang werden wir im Folgenden erläutern. Zunächst müssen wir aber klären, was eine Skala überhaupt ist. Das Tonsystem, das heutzutage in der westlichen Musik verwendet wird, besteht aus zwölf verschiedenen Tönen: Dies sind die sieben Stammtöne C, D, E, F, G, A, H sowie die fünf Töne $C \sharp / D b, D \sharp / E b, F \sharp / G b, G \sharp / A b, A \sharp / B$, die als Erniedrigung/Erhöhung der sieben Stammtöne auftreten (Abbildung 1).

Aus mathematischer Sicht wollen wir nicht zwischen den Tönen $C \sharp$ und $D b$ unterscheiden und werden daher im Folgenden immer die $\sharp$-Konvention benutzen. Eine Skala ist nun einfach definiert als eine Teilmenge von $\{C, C \sharp, D, D \sharp, E, F, F \sharp, G, G \sharp, A, A \sharp, H\}$. Als Beispiel betrachten wir die C-Dur-Skala in unserer Teilmengennotation:

$$
\{C, D, E, F, G, A, H\} \text {. }
$$

Die zwölf Töne haben eine zyklische Ordnung. So folgt beispielsweise in der C-Dur-Skala nach dem $H$ wieder das $C$ (siehe Abbildung 2). Diese zyklische Anordnung erlaubt es uns, einen Abstand zwischen zwei Tönen $t_{1}, t_{2} \in\{C, C \sharp, D, D \sharp, E, F, F \sharp, G, G \sharp, A, A \sharp, H\}$ zu definieren: Zum einen können wir die Distanz von $t_{1}$ nach $t_{2}$ im Uhrzeigersinn betrachten und zum anderen die Distanz von $t_{2}$ nach $t_{1}$ ebenfalls im Uhrzeigersinn. Der Abstand zwischen $t_{1}$ und $t_{2}$ ist dann als Minimum dieser beiden Distanzen definiert. Betrachten wir die Töne $A$ und $C$ : Die Distanz von $A$ nach $C$ ist drei und die Distanz von $C$ nach $A$ ist neun, sodass diese beiden Töne den Abstand drei haben. Die Abstände zwischen aufeinanderfolgenden Tönen einer Skala in der zyklischen Ordnung bezeichnen wir als die Intervallfolge der Skala. Wie wir in Abbildung 2 sehen, hat die C-Dur-Skala die Intervallfolge 2-2-1-2-2-2-1.

Die zyklische Ordnung der zwölf Töne impliziert, dass die Intervalle einer Skala ebenfalls zyklisch angeordnet sind. Also können wir zum Beispiel ebenso sagen, dass die C-DurSkala die Intervallfolge 2-1-2-2-2-1-2 hat. Aus musikalischer Sicht würde man sagen, man startet die Dur-Skala von einem anderen Grundton als $C$ ausgehend - in diesem Fall von $D$. Daraus ergeben sich die in der Musik häufig verwendeten Kirchentonarten, D-Dorisch im konkreten Fall.

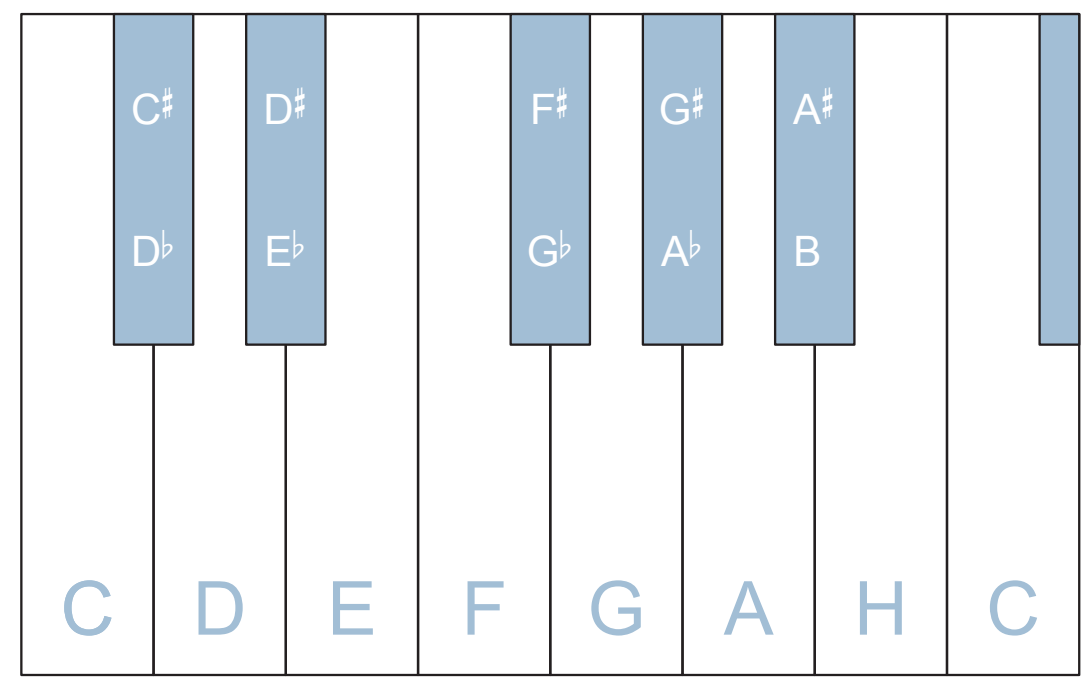

Abbildung 1. Westliches Tonsystem der Neuzeit mit zwölf Tönen 


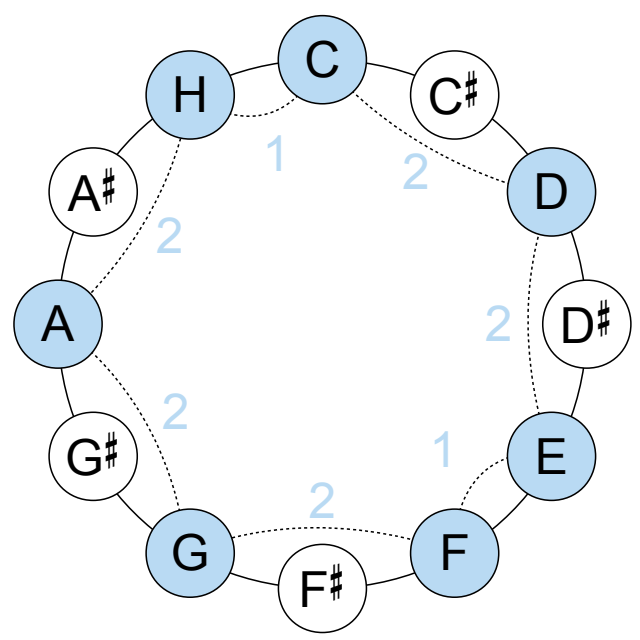

Abbildung 2. Zyklische Ordnung der zwölf Töne Blau markiert: C-Dur-Skala mit Intervallfolge

Mathematisch gesehen würden wir die Skalen C-Jonisch, D-Dorisch, E-Phrygisch, F-Lydisch, G-Mixolydisch, A-Äolisch (natürliches a-Moll) und $H$-Lokrisch sämtlich miteinander identifizieren, da sie die gleiche Intervallfolge besitzen. In der Musik spricht man auch von den Modi der Dur-Skala. Sie sind von Bedeutung im modalen Jazz. Da es zwölf DurSkalen und daher $12 \cdot 7=84$ verschiedene Kirchentonarten gibt, wird im erwähnten Buch von Deuker [1] der didaktische Ansatz vertreten, sich auf die Betrachtung der DurSkalen zu beschränken und darauf zu verzichten, alle diese Kirchentonarten lernen zu lassen, obwohl dies heute noch in der weit verbreiteten Skalentheorie üblich ist. Ein moderner Vertreter letzteren Ansatzes ist zum Beispiel Frank Sikora [6].

Ein Intervall der Länge eins wird als Halbton(schritt) und ein Intervall der Länge zwei als Ganzton(schritt) bezeichnet. Die Skala, deren Intervalle zwölf Halbtöne sind (und die daher alle zwölf Töne enthält), wird chromatische Skala genannt. Daher nennen wir eine Skala, deren Intervallfolge keine zwei aufeinanderfolgenden Halbtonschritte enthält, nicht-chromatisch. Dies ist äquivalent dazu, dass die Skala keine drei aufeinanderfolgenden Töne in der zyklischen Ordnung von Abbildung 2 enthält. Die C-Dur-Skala ist ein Beispiel für solch eine nicht-chromatische Skala. Ein Gegenbeispiel ist die Skala

$$
\{C, C \sharp, E, F, G, A, H\},
$$

mit der Intervallfolge 1-3-1-2-2-2-1. Man kann mit einer gewissen Berechtigung die nicht-chromatischen Skalen als „Grundfarben“ in der Musik ansehen, während (teil-) chromatische Skalen mit mindestens zwei aufeinanderfolgenden Halbtonschritten dann „Mischfarben“ wären. Dies ist im erwähnten Buch [1] der Ansatz, in dem versucht wird, den Raum aller Skalen systematisch zu ordnen.

Wir betrachten hier aber nicht-chromatische Skalen aus der mathematischen Perspektive. Die nicht-chromatischen Skalen bilden einen Simplizialkomplex. Solch ein Simpli- zialkomplex ist auf einer Grundmenge $\mathcal{G}$ definiert als eine Menge $\mathcal{K}$ von endlichen Teilmengen von $\mathcal{G}$, sodass für jede Menge $M$ in $\mathcal{K}$ und jede Teilmenge $T$ von $M$ gilt, dass $T$ ebenfalls in $\mathcal{K}$ ist. Als Beispiel wählen wir die Grundmenge $\mathcal{G}:=\{0,1,2\}$. Die Menge $\mathcal{K}_{1}:=\{\{0,1\},\{2\}\}$ ist kein Simplizialkomplex, da $\{0\}$ eine Teilmenge von $\{0,1\}$ ist, aber trotzdem nicht in $\mathcal{K}_{1}$ enthalten ist. Wir können $\mathcal{K}_{1}$ aber zu einem Simplizialkomplex wie folgt erweitern: $\mathcal{K}_{2}:=\{\{0,1\},\{0\},\{1\},\{2\}, \emptyset\}$.

Die Menge aller nicht-chromatischen Skalen ist ein Simplizialkomplex auf der Grundmenge der zwölf Töne $\mathcal{G}:=$ $\{C, C \sharp, D, D \sharp, E, F, F \sharp, G, G \sharp, A, A \sharp, H\}$. Das liegt daran, dass beim Entfernen von Tönen von einer nicht-chromatischen Skala die Skala stets nicht-chromatisch bleibt. Diesen Komplex der nicht-chromatischen Skalen bezeichnen wir im Folgenden mit $\mathcal{K}_{N C}$. Mit anderen Worten können wir $\mathcal{K}_{N C}$ definieren als die Menge aller Teilmengen der zwölf Töne in Abbildung 2, die keine drei aufeinanderfolgenden Töne enthalten. Im Folgenden wollen wir drei Fragen über $\mathcal{K}_{N C}$ beantworten, die Mathematiker typischerweise an einen Simplizialkomplex stellen, und wir werden sehen, dass diese Fragen ebenfalls musikalisch relevant sind.

\section{$f$-Vektor}

Wir können uns die $n$-elementigen Mengen in einem Simplizialkomplex geometrisch als $(n-1)$-dimensionale Objekte vorstellen. So entspricht eine 1-elementige Menge einem Punkt, eine 2-elementige Menge einem Liniensegment, eine 3-elementige Menge einem Dreieck, eine 4-elementige Menge einem Tetraeder, usw. Dies erlaubt es uns auch, Simplizialkomplexe zeichnerisch darzustellen (siehe Abbildung 3 für eine Darstellung von obigem Beispiel $\mathcal{K}_{2}$ ). Die Zeichnung ist allerdings rein schematisch zu verstehen; beispielsweise kommt es hier nicht auf Einbettbarkeit an. 


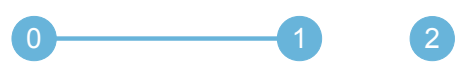

Abbildung $3 \cdot \mathcal{K}_{2}$

Die höherdimensionale Verallgemeinerung eines Tetraeders wird Simplex genannt. Daher stammt auch der Name Simplizialkomplex. Sei nun $\mathcal{K}$ ein Simplizialkomplex und $n$ eine natürliche Zahl. Dann bezeichnen wir mit $f_{n}$ die Anzahl der $n$-dimensionalen Simplizes in $\mathcal{K}$ (also die Anzahl der $(n+1)$-elementigen Mengen in $\mathcal{K}$ ). Die leere Menge $\emptyset$, die in jedem Simplizialkomplex enthalten ist, betrachten wir als -1-dimensionalen Simplex und daher setzen wir $f_{-1}=1$. Die Liste $\left(f_{-1}, f_{0}, f_{1}, f_{2}, f_{3}, \ldots\right)$ aller dieser Zahlen wird $f$-Vektor von $\mathcal{K}$ genannt. Zum Beispiel ist $(1,3,1)$ der $f$-Vektor von $\mathcal{K}_{2}$.

Für unseren Simplizialkomplex $\mathcal{K}_{N C}$ bedeutet die Frage nach dem $f$-Vektor, wie viele nicht-chromatische Skalen es mit $0,1,2,3, \ldots, 12$ Tönen gibt. Dies ist zum Beispiel interessant, wenn man nicht-chromatisch improvisieren möchte. Insbesondere möchte man dann wissen, wie viele Töne man dabei maximal verwenden kann. Zudem gibt es mehrere Improvisationsansätze, die auf Pentatoniken - das sind Skalen mit fünf Tönen - aufbauen, und daher ist die Anzahl der nicht-chromatischen Pentatoniken relevant. Wir berechneten $\operatorname{den} f$-Vektor von $\mathcal{K}_{N C}$ mit der Software polymake [2]:

$$
\left.\begin{array}{ccccccccc}
(1, & 12, & 66, & 208, & 399, & 456, & 282, & 72, & 3
\end{array}\right) .
$$

Dies bedeutet insbesondere, dass es keine nicht-chromatischen Skalen mit neun oder mehr Tönen gibt, und dass es genau drei solche Skalen mit acht Tönen gibt. Diese drei Skalen werden wir im nächsten Abschnitt noch genauer betrachten. Außerdem können wir ablesen, dass es 456 nichtchromatische Pentatoniken gibt.

\section{Facetten}

Die inklusionsmaximalen Mengen in einem Simplizialkomplex $\mathcal{K}$ werden auch Facetten genannt. Dies sind also alle Mengen in $\mathcal{K}$, die nicht in einer größeren Menge aus $\mathcal{K}$ enthalten sind. Im obigem Beispiel $\mathcal{K}_{2}$ sind die Facetten die Mengen $\{0,1\}$ und $\{2\}$.

In der Sprache der Musik ausgedrückt wollen wir wissen, wie viele nicht-chromatische Skalen es gibt, zu denen man keinen weiteren Ton hinzufügen kann, ohne zwei aufeinanderfolgende Halbtonschritte zu erzeugen. Ein Beispiel für eine solche Skala ist die C-Dur-Skala. Dies ist ebenfalls beim Improvisieren ein wichtiges Konzept: Man muss sich nur diese maximalen nicht-chromatischen Skalen merken, da alle anderen nicht-chromatischen Skalen Teilmengen der maximalen sind. Das ist auch der Grund, warum sich das erwähnte Buch von Deuker [1] detailliert mit diesen Skalen beschäftigt. Er zeigt sogar im zweiten Kapitel, dass es genau 57 maximale nicht-chromatische Skalen gibt, und dass alle diese Skalen immense Bedeutung in der Musikgeschichte haben. Es ist bemerkenswert, dass diese Skalen also sowohl in der Musik bedeutsam als auch in der mathematischen Formulierung (als Facetten von $\mathcal{K}_{N C}$ ) sichtbar sind.

Im Folgenden wollen wir ebenfalls die 57 Facetten von $\mathcal{K}_{N C}$ auflisten. Wir sehen, dass die 57 maximalen nichtchromatischen Skalen nur sieben verschiedene Intervallfolgen haben. Zur Intervallfolge in der ersten Zeile der Tabelle 1 gehören drei verschiedene Skalen mit jeweils acht Tönen. Diese drei Skalen sind genau jene, die wir auch bei der Berechnung des $f$-Vektors erhalten haben. Außerdem sehen wir 48 maximale nicht-chromatische Skalen, die aus sieben Tönen bestehen. Von diesen gibt es vier verschiedene Typen, je nach Intervallfolge. Im berechneten $f$-Vektor können wir sehen, dass es insgesamt $72=48+24$ nicht-chromatische Skalen mit sieben Tönen gibt. Die übrigen $24=3 \cdot 8$ Skalen sind Teilmengen der drei achttönigen Skalen in Abbildung 4, die jeweils acht siebentönige Teilmengen haben. Weiterhin gibt es sechs maximale nicht-chromatische Skalen mit sechs Tönen. Insbesondere bedeutet dies für unseren Simplizialkomplex $\mathcal{K}_{N C}$, dass seine Facetten verschieden groß sind. In der Mathematik sagen wir dazu, dass $\mathcal{K}_{N C}$ nicht pur ist.

Nun wollen wir aber auf Namen und Bedeutung der 57 Facetten in der Musik eingehen.

$2-1-2-1-2-1-2-1$ :

$\mathrm{Zu}$ dieser Intervallfolge finden wir die drei verschiedenen Skalen in Abbildung 4. Diese werden verminderte Skalen genannt und sind in der Musik die gängigsten Skalen mit acht Tönen. Heutzutage werden sie häufig im Jazz benutzt.

Tabelle 1

\begin{tabular}{clcl}
\hline Anzahl Töne & Intervallfolge & Anzahl Skalen & Name \\
\hline 8 & $2-1-2-1-2-1-2-1$ & 3 & vermindert \\
7 & $2-2-1-2-2-2-1$ & 12 & Dur \\
7 & $2-1-2-2-2-2-1$ & 12 & melodisch Moll \\
7 & $2-1-2-2-1-3-1$ & 12 & harmonisch Moll \\
7 & $2-2-1-2-1-3-1$ & 12 & harmonisch Dur \\
6 & $2-2-2-2-2-2$ & 2 & Ganzton \\
6 & $1-3-1-3-1-3$ & 4 & übermäßig \\
\hline
\end{tabular}




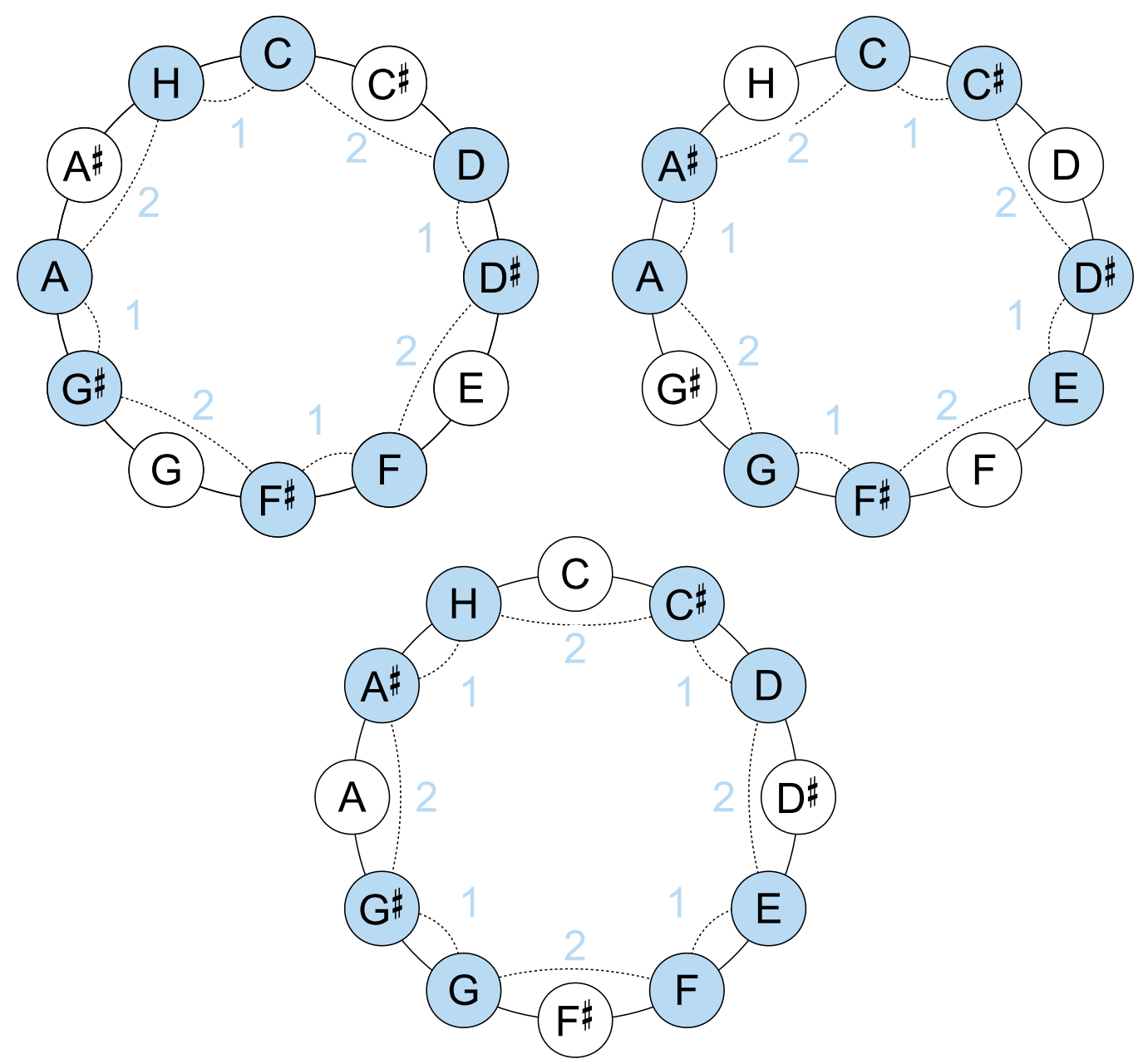

Abbildung 4. Die drei verminderten Skalen

Diese Skalen werden zum Beispiel im Jazz-Lehrbuch [7] ausführlich behandelt. Um das Jahr 1900 herum wurden verminderte Skalen aber auch häufig in Russland, insbesondere vom Komponisten Rimski-Korsakov, eingesetzt sowie in den Niederlanden von Willem Pijper, weswegen die Skalen in diesen Ländern auch Rimski-Korsakov-Skala oder Pijper-Skala genannt werden.

\section{$2-2-1-2-2-2-1:$}

Diese Intervallfolge entspricht der wohlbekannten DurSkala. Als Anfangsbeispiel hatten wir bereits die C-DurSkala betrachtet, aber alle zwölf Möglichkeiten einen Anfangston zu wählen, ergeben zwölf verschiedene Dur-Skalen. Diese sind die am meisten verwendeten Skalen in der westlichen Musik. Wir haben bereits erwähnt, dass obige Intervallfolge ebenfalls den natürlichen Moll-Skalen entspricht. So hat etwa die C-Dur-Skala genau die gleichen Töne wie die natürliche a-Moll-Skala. Spricht man von Moll-Skalen, so wird die Intervallfolge in der Regel als 2-1-2-2-1-2-2 geschrieben. Nach den Dur-Skalen ist diese Variante des Molls die zweithäufigste in der abendländischen Musik.
$2-1-2-2-2-2-1$ :

Dies sind die melodischen Moll-Skalen. Von diesen gibt es ebenfalls zwölf verschiedene, je nach Anfangston. Die melodischen Moll-Skalen sind weit verbreitet, von frühen Kompositionen - insbesondere für Vokalmusik - bis hin zu modernem Pop und Rock. Es gibt eine in Büchern über Harmonielehre weit verbreitete Regel, dass melodisch Moll aufwärts gespielt die oben definierte Skala darstellt, während man abwärts gespielt die natürliche Moll-Skala mit dem gleichen Grundton verwenden soll. Aus mathematischer Sicht ist diese Regel uninteressant und Deuker [1] vertritt die Auffassung, dass sie auch in musikalischer Hinsicht unsinnig ist, da konkrete Melodien selten einfach linear aboder aufsteigend sind.

$2-1-2-2-1-3-1$ :

$\mathrm{Zu}$ dieser Intervallfolge gehören die harmonischen MollSkalen. Alle zwölf Anfangstöne ergeben zwölf verschiedene solche Skalen. Im Gegensatz zum melodischen Moll werden die harmonischen Moll-Skalen vor allem zur Bildung von Akkorden verwendet, aber insbesondere bei Mozart oder auch Schubert treten sie ebenfalls in Melodien auf. 
$2-2-1-2-1-3-1$ :

Von diesem letzten Typ siebentöniger maximaler nichtchromatischer Skalen gibt es wieder zwölf verschiedene. Diese werden harmonische Dur-Skalen genannt. Sie werden als eine Mischung von Dur- und harmonischen Moll-Skalen empfunden und am häufigsten im Jazz verwendet.

$2-2-2-2-2-2$ :

Dies ist die Ganztonskala. Aufgrund ihrer hohen Symmetrie gibt es von ihr nur zwei verschiedene Ausführungen, je nach Anfangston. Da es allerdings keine Halbtonschritte gibt, ist der Anfangston eine relativ willkürliche Festlegung und es gibt keinen hörbaren Grundton der Skala. Dadurch wird die Wirkung der Skala oft als schwebend bezeichnet. In moderner westlicher Musik findet sie außerhalb des Jazz nur extrem selten Verwendung, aber im Impressionismus insbesondere bei Debussy - spielte sie eine große Rolle und wurde zuvor von Franz Liszt und Rimski-Korsakow eingesetzt.

$1-3-1-3-1-3$ :

Hierzu gehören die übermäßigen Skalen. Analog zum Fall der verminderten Skalen (siehe Abbildung 4) gibt es hiervon vier verschiedene Skalen. Diese wurden bereits von Franz Liszt benutzt, tauchten aber vermehrt im 20. Jahrhundert bei Komponisten wie Béla Bartók oder Arnold Schönberg sowie im Jazz auf. Es gibt sogar ein Jazz-Lehrbuch, das sich explizit diesen Skalen widmet [5].

Zum Schluss wollen wir noch herleiten, wieso es für die Intervallfolge $\mathcal{I}$ einer maximalen nicht-chromatischen Skala genau die sieben verschiedenen Möglichkeiten aus der obigen Tabelle gibt.

Beobachtung 1:

I kann kein Intervall der Länge vier oder mehr enthalten. Würde $\mathcal{I}$ ein solches Intervall haben $-z$. B. zwischen den Tönen $C$ und $E$-, so könnten wir einen Ton in der Mitte dieses Intervalls ( $D$ im Beispiel) hinzufügen und die Skala bliebe nicht-chromatisch.

\section{Beobachtung 2:}

Wenn $\mathcal{I}$ ein Intervall der Länge drei enthält, so muss dieses als linken und rechten Nachbarn in $\mathcal{I}$ jeweils einen Halbton haben. In anderen Worten: Wenn 3 in $\mathcal{I}$ enthalten ist, so muss $\mathcal{I}$ die Folge $1-3-1$ enthalten. Würde $\mathcal{I}$ nämlich die Folge $3-2$ enthalten - z. B. wenn die Skala die Töne $C, D \sharp$ und $F$ enthielte -, so könnten wir einen Ton aus dem Intervall der Länge drei (im Beispiel D) zur Skala hinzufügen, sodass diese weiterhin nicht-chromatisch wäre.

Mit diesen zwei Beobachtungen können wir nun ein paar Fälle unterscheiden, um alle sieben Möglichkeiten für I herauszufinden.

Fall 1:

I enthält eine drei und somit - nach Beobachtung 2 - die Folge $1-3-1$. Nun können wir diese Folge entweder mit einer drei oder mit einer zwei fortsetzen. Falls wir die Folge mit einer drei fortsetzen, so muss $\mathcal{I}$ die Folge $1-3-1-3-1$ enthalten. Da sich alle Zahlen in $\mathcal{I}$ zu zwölf aufsummieren müssen, gibt es nun nur noch eine Möglichkeit, die Folge zu einer Intervallfolge einer nicht-chromatischen Skala zu erweitern:

$$
1-3-1-3-1-3 .
$$

Hätten wir die Folge 1-3-1 nach links mit einer drei erweitert, wären wir auf die gleiche Intervallfolge gekommen. Also müssen wir nun nur noch den Fall betrachten, dass I die Folge 2-1-3-1-2 enthält. Nach Beobachtung 2 müssen wir diese Folge mit einer zwei und einer eins vervollständigen, wofür es zwei Möglichkeiten gibt:

$$
\begin{aligned}
& 2-2-1-2-1-3-1 \\
& 2-1-2-2-1-3-1
\end{aligned}
$$

Fall 2:

I enthält keine drei und besteht somit nur aus Einsen und Zweien. Da alle Zahlen in $\mathcal{I}$ in Summe zwölf ergeben müssen, ist die Anzahl der Einsen in $\mathcal{I}$ gerade. Falls $\mathcal{I}$ keine Einsen enthält, so sieht $\mathcal{I}$ wie folgt aus:

$$
2-2-2-2-2-2 \text {. }
$$

Falls $\mathcal{I}$ zwei Einsen enthält, so gibt es nur zwei verschiedene Optionen, diese Einsen anzuordnen:

$$
\begin{aligned}
& 2-1-2-2-2-2-1 \\
& 2-2-1-2-2-2-1
\end{aligned}
$$

Die weiteren Möglichkeiten 2-2-2-1-2-2-1 und 2-2-2-2-1-2-1 sind nämlich aufgrund der zyklischen Anordnung identisch mit den beiden obigen Folgen. Enthält $\mathcal{I}$ vier Einsen, so muss $\mathcal{I}$ wie folgt aussehen:

$$
2-1-2-1-2-1-2-1 \text {. }
$$

Dies zeigt auch, dass $\mathcal{I}$ nicht mehr als vier Einsen enthalten kann.

\section{Topologie}

Im mathematischen Teilgebiet der Topologie untersucht man, welche Eigenschaften von Räumen unter stetigen Verformungen erhalten bleiben. Dabei heißt eine Verformung stetig, falls sie - kurz gefasst - weder das Zerschneiden noch das Zusammenkleben von Raumteilen benötigt. Typische Beispiele für stetige Verformungen sind das Dehnen und Biegen von Räumen. So kann man eine Kugel stetig zu einem Würfel verformen. Da man diese Verformung ebenfalls auf stetige Art und Weise wieder rückgängig machen kann, sagt man, dass Kugel und Würfel homöomorph zueinander sind. Eine Tasse mit einem Henkel ist homöomorph zu einem Donut mit einem Loch. Allerdings ist solch ein Donut nicht homöomorph zur Kugel, da man sein Loch nicht stetig füllen kann. Daher ist die Anzahl der Löcher ein Beispiel für eine Eigenschaft, die homöomorphe Räume gemeinsam haben.

Die Idee von Homöomorphie kann zu Homotopieäquivalenz verallgemeinert werden, welche ebenfalls die Anzahl der Löcher erhält. Seien $X$ und $Y$ zwei Räume mit zwei stetigen Abbildungen $g: X \rightarrow Y$ und $f: Y \rightarrow X$. Falls die 
Verknüpfungen $f \circ g: X \rightarrow X$ und $g \circ f: Y \rightarrow Y$ die Identitäten auf $X$ bzw. $Y$ sind (d.h. $f(g(x))=x$ für alle $x \in X$ und $g(f(y))=y$ für alle $y \in Y)$, dann sind $X$ und $Y$ homöomorph zueinander. Falls die Abbildung $f \circ g$ stetig zur Identität auf $X$ und $g \circ f$ stetig zur Identität auf $Y$ verformt werden kann, so sagen wir, dass $X$ und $Y$ homotopieäquivalent sind. Daher ist jeder Homöomorphismus eine Homotopieäquivalenz. Das Aufdicken und Zusammenquetschen von Räumen sind Beispiele für Homotopieäquivalenzen, die keine Homöomorphismen sind. Zum Beispiel sind eine Kugel und ein Punkt homotopieäquivalent, aber nicht homöomorph.

\section{Löcher in Simplizialkomplexen}

Auch in einem Simplizialkomplex $\mathcal{K}$ kann man Löcher zählen. Dazu bestimmen wir die (reduzierte) simpliziale Homologie des Komplexes. Wir geben nun eine kurze Einführung in dieses Thema und verweisen den interessierten Leser auf [4]. Wie in der Beschreibung des $f$-Vektors betrachten wir alle $n$-dimensionalen Simplizes in $\mathcal{K}$. Eine formale Summe über diese Simplizes bezeichnet einfach eine Summe von diesen Simplizes mit rationalen Koeffizienten, die man hinschreibt, ohne sie auszuwerten. Dann bezeichnen wir mit $C_{n}(\mathcal{K})$ die Menge aller solchen formalen Summen. Diese ist ein Vektorraum über den rationalen Zahlen $\mathbb{Q}$. In unserem Beispielkomplex $\mathcal{K}_{2}$ haben wir unter anderem folgende formalen Summen:

$$
\frac{3}{2}\{0,1\} \in C_{1}\left(\mathcal{K}_{2}\right), 2\{0\}-\{2\} \in C_{0}\left(\mathcal{K}_{2}\right),-\emptyset \in C_{-1}\left(\mathcal{K}_{2}\right) .
$$

Die Randabbildung bildet ein $n$-dimensionales Simplex auf seinen $(n-1)$-dimensionalen Rand ab. Zum Beispiel bildet sie ein Dreieck auf seine drei begrenzenden Liniensegmente und einen Tetraeder auf seine vier Flächen (welche Dreiecke sind) ab. Formal wird die Randabbildung $\partial_{n}: C_{n}(\mathcal{K}) \rightarrow C_{n-1}(\mathcal{K})$ dadurch definiert, dass sie jede $(n+1)$ elementige Menge in $\mathcal{K}$ auf eine alternierende Summe ihrer $n$-elementigen Teilmengen schickt. Dabei werden die Elemente einer $(n+1)$-elementigen Menge nacheinander in einer zuvor fixierten Reihenfolge weggelassen. In unserem Beispiel $\mathcal{K}_{2}$ bedeutet dies:

$$
\begin{aligned}
\partial_{1}(\{0,1\}) & =\{1\}-\{0\}, \\
\partial_{0}(\{0\}) & =\partial_{0}(\{1\})=\partial_{0}(\{2\})=\emptyset .
\end{aligned}
$$

Die formalen Summen in $C_{n}(\mathcal{K})$, die von der Randabbildung auf 0 geschickt werden, heißen $n$-Zykel. Die Menge aller solchen Zykel ist ein Vektorraum, welchen wir mit $Z_{n}(\mathcal{K})$ bezeichnen. Das Bild $\partial_{n+1}(\Sigma)$ einer formalen Summe $\sum \in C_{n+1}(\mathcal{K})$ nennen wir einen $n$-Rand. Die Menge aller dieser Ränder ist ebenfalls ein Vektorraum, welchen wir mit $R_{n}(\mathcal{K})$ bezeichnen. Nun kann man direkt nachrechnen, dass jeder $n$-Rand ein $n$-Zykel ist, d. h. $R_{n}(\mathcal{K}) \subseteq Z_{n}(\mathcal{K})$. Wir sagen, dass zwei $n$-Zykel äquivalent sind, wenn ihre Differenz ein $n$ Rand ist. Die Äquivalenzklasse eines $n$-Zykels $\zeta$ ist die Menge aller $n$-Zykel, die zu $\zeta$ äquivalent sind. Wir bezeichnen mit $H_{n}(\mathcal{K})$ die Menge aller solchen Äquivalenzklassen, welche auch ein Vektorraum ist und $n$-te Homologiegruppe von $\mathcal{K}$ genannt wird.

Im obigen Beispiel $\mathcal{K}_{2}$ sind die 0 -Zykel alle formalen Summen der Form $a\{0\}+b\{1\}+c\{2\}$, wobei $a, b, c$ rationale Zahlen mit $a+b+c=0$ sind. Die 0-Ränder sehen so aus: $d\{0\}-d\{1\}$ mit $d \in \mathbb{Q}$. Also besteht die Äquivalenzklasse eines 0-Zykels $a\{0\}+b\{1\}+c\{2\}$ aus allen 0-Zykeln, die den gleichen Koeffizienten $c$ vor $\{2\}$ haben. Daher ist solch eine Äquivalenzklasse durch den Koeffizienten $c$ eindeutig bestimmt. Da $c$ eine beliebige rationale Zahl ist, sagen wir dass $H_{0}\left(\mathcal{K}_{2}\right)$ isomorph zu $\mathbb{Q}$ ist. Der einzige 1 -Zykel von $\mathcal{K}_{2}$ ist 0 (d.h. die formale Summe deren Koeffizienten 0 sind). Weil $\mathcal{K}_{2}$ keine 3-elementige Menge enthält, können wir sagen, dass der einzige 1 -Rand von $\mathcal{K}_{2}$ ebenfalls 0 ist. Demnach enthält $H_{1}\left(\mathcal{K}_{2}\right)$ nur die 0 .

Betrachten wir die Darstellung von $\mathcal{K}_{2}$ in Abbildung 3, so sehen wir, dass $\mathcal{K}_{2}$ nicht zusammenhängend ist. Dies bedeutet, dass man $\mathcal{K}_{2}$ nicht zeichnen kann, ohne den Stift abzusetzen. Stattdessen hat $\mathcal{K}_{2}$ zwei Zusammenhangskomponenten. Die Anzahl dieser Komponenten können wir auch von der ersten Homologiegruppe ablesen: Für jeden Simplizialkomplex $\mathcal{K}$ gilt, dass $H_{0}(\mathcal{K})$ isomorph zum Vektorraum $\mathbb{Q}^{k-1}$ ist, wobei $k$ die Anzahl der Zusammenhangskomponenten von $\mathcal{K}$ ist. Wir verwenden die Konvention, dass $\mathbb{Q}^{0}=\{0\}$ ist.

Nun können wir aber nicht nur die Zusammenhangskomponenten eines Simplizialkomplexes mittels Homologie zählen, sondern auch die Anzahl der Löcher. Dies erklären wir am Beispiel eines ausgefüllten und eines nichtausgefüllten Dreiecks:

$$
\begin{aligned}
& \mathcal{K}_{\Delta}:=\{\{0,1\},\{1,2\},\{0,2\},\{0\},\{1\},\{2\}, \emptyset\}, \\
& \mathcal{K}_{\Delta}:=\{\{0,1,2\},\{0,1\},\{1,2\},\{0,2\},\{0\},\{1\},\{2\}, \emptyset\} .
\end{aligned}
$$

Abbildung 5 ist die Darstellung dieser beiden Komplexe in der Ebene.
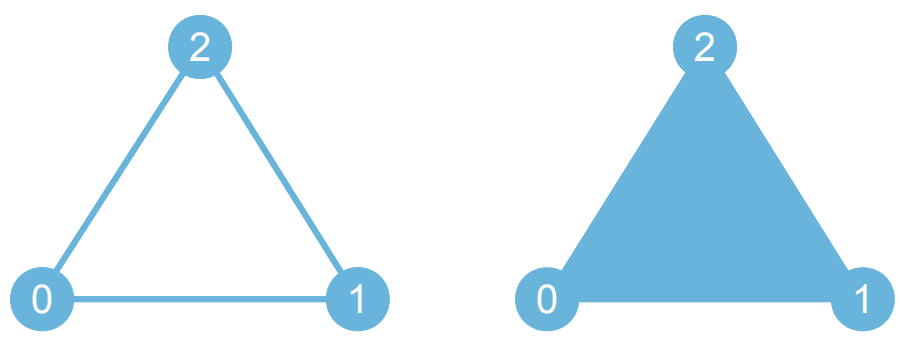

Abbildung $5 . \mathcal{K}_{\Delta}$ und $\mathcal{K}_{\Delta}$ 
Da beide Dreiecke zusammenhängend sind, wissen wir, dass $H_{0}\left(\mathcal{K}_{\Delta}\right)$ und $H_{0}\left(\mathcal{K}_{\mathbf{\Delta}}\right)$ zu $\{0\}$ isomorph sein müssen. Dies können wir auch direkt nachrechnen: Sowohl für $\mathcal{K}_{\Delta}$ als auch $\mathcal{K}_{\mathbf{\Delta}}$ sind die ersten zwei Randabbildungen gleich:

$$
\begin{aligned}
& \partial_{1}(\{0,1\})=\{1\}-\{0\}, \\
& \partial_{1}(\{1,2\})=\{2\}-\{1\}, \\
& \partial_{1}(\{0,2\})=\{2\}-\{0\}, \\
\partial_{0}(\{0\})= & \partial_{0}(\{1\})=\partial_{0}(\{2\})=\emptyset .
\end{aligned}
$$

Für beide Simplizialkomplexe sind daher alle 0-Zykel von der Form $a\{0\}+b\{1\}+c\{2\}$, wobei $a, b, c$ rationale Zahlen mit $a+b+c=0$ sind. Die 0-Ränder haben die Form

$$
\begin{aligned}
d(\{1\}-\{0\})+e(\{2\}-\{1\})+f(\{2\}-\{0\}) \\
=(-d-f)\{0\}+(d-e)\{1\}+(e+f)\{2\}
\end{aligned}
$$

mit $d, e, f \in \mathbb{Q}$. Daran kann man sehen, dass jeder 0-Zykel ein 0 -Rand ist. Also gibt es nur eine Äquivalenzklasse von 0 -Zykeln und $H_{0}\left(\mathcal{K}_{\Delta}\right)=H_{0}\left(\mathcal{K}_{\mathbf{\Delta}}\right)$ ist eine 1-elementige Menge und daher insbesondere isomorph zu $\{0\}$, wie wir es oben behauptet haben.

Die 1-Zykel von $\mathcal{K}_{\Delta}$ und $\mathcal{K}_{\mathbf{\Delta}}$ sind von der Form $d\{0,1\}+$ $d\{1,2\}-d\{0,2\}$. Die Randabbildung $\partial_{2}$ des ausgefüllten Dreiecks $\mathcal{K}_{\mathbf{\Delta}}$ bildet $\{0,1,2\}$ auf $\{1,2\}-\{0,2\}+\{0,1\}$ ab. Deshalb sehen die 1-Ränder von $\mathcal{K}_{\mathbf{\Delta}}$ genauso aus wie die 1-Zykel und $H_{1}\left(\mathcal{K}_{\mathbf{\Delta}}\right)$ enthält nur eine Äquivalenzklasse. Also ist $H_{1}\left(\mathcal{K}_{\mathbf{\Delta}}\right)$ isomorph zu $\mathbb{Q}^{0}$. Da das nicht-ausgefüllte Dreieck $\mathcal{K}_{\Delta}$ keine 3-elementigen Mengen enthält, ist der einzige 1-Rand von $\mathcal{K}_{\Delta}$ gleich 0 . Verschiedene 1 -Zykel von $\mathcal{K}_{\Delta}$ können also nie äquivalent sein. Weil der Koeffizient $d$ der 1 -Zykel eine beliebige rationale Zahl ist, ist $H_{1}\left(\mathcal{K}_{\Delta}\right)$ isomorph zu $\mathbb{Q}^{1}$. Das Dreieck $\mathcal{K}_{\Delta}$ hat ein (eindimensionales) Loch, wohingegen $\mathcal{K}_{\mathbf{\Delta}}$ keine Löcher hat. Wir sehen also, dass für beide Dreiecke gilt, dass die Dimension von $H_{1}\left(\mathcal{K}_{\Delta}\right)$ bzw. $H_{1}\left(\mathcal{K}_{\mathbf{\Delta}}\right)$ die Anzahl der eindimensionalen Löcher von $\mathcal{K}_{\Delta}$ bzw. $\mathcal{K}_{\mathbf{\Delta}}$ ist.

Wir sehen auch in Abbildung 3, dass $\mathcal{K}_{2}$ keine eindimensionalen Löcher hat, was dazu passt, dass $H_{1}\left(\mathcal{K}_{2}\right)=\{0\}$ ist. Diese Idee können wir verallgemeinern: Anschaulich gesprochen zählt die Dimension von $H_{n}(\mathcal{K})$ die $n$-dimensionalen Löcher eines Simplizialkomplexes $\mathcal{K}$.

Löcher in $\mathcal{K}_{N C}$

Nun stellt sich die Frage, wie viele Löcher in welchen Dimensionen der Komplex der nicht-chromatischen Skalen hat und was dies musikalisch bedeutet. Mithilfe der Software polymake ermitteln wir, dass $H_{5}\left(\mathcal{K}_{N C}\right)$ isomorph zu $\mathbb{Q}^{3}$ ist und dass alle anderen Homologiegruppen nur $\{0\}$ sind. Daraus können wir weiterhin schlussfolgern, dass $\mathcal{K}_{N C}$ drei 5-dimensionale Löcher hat. Die Software polymake gibt uns sogar für jedes der drei Löcher eine Basis der Homologie, d. h eine Menge von sechstönigen Skalen, die das Loch abtrennen. Zudem können wir mit polymake zeigen, dass die Vereinigung der sechstönigen Skalen, die solch ein Loch abtrennen, homöomorph zur 5-Sphäre ist. Die n-Sphäre ist der Rand einer $(n+1)$-dimensionalen Kugel. So ist beispielsweise das nicht-ausgefüllte Dreieck in Abbildung 5 homöomorph zur 1-Sphäre, welche einfach nur ein Kreisrand ist. Kurz gefasst kann man sich den Komplex der nicht-chromatischen Skalen daher als aus drei 5-Sphären zusammengesetzt vorstellen.

Die 5-Sphären bestehen wie erwähnt aus sechstönigen Skalen. Zusätzlich liegen alle Teilskalen einer solchen sechstönigen Skala ebenfalls auf der jeweiligen Sphäre, so wie das nicht-ausgefüllte Dreieck in Abbildung 5 aus 2-elementigen Mengen besteht, deren 1-elementige Teilmengen aber als Punkte auf dem Dreieck liegen. Nun haben wir aber auch sieben- und achttönige Skalen in $\mathcal{K}_{N C}$. Wieso diese im topologischen Bild der drei Sphären keine Rolle spielen, erklären wir mit sogenannten Kollapsen (siehe etwa [3]). Immer dann, wenn ein Simplizialkomplex $\mathcal{K}$ eine Facette $F$ mit einer Teilmenge $M \subseteq F$ hat, sodass die folgenden zwei Eigenschaften gelten:

1. $M$ enthält genau ein Element weniger als $F$,

2. $F$ ist die einzige Facette, die $M$ enthält,

können wir $F$ und $M$ aus dem Komplex $\mathcal{K}$ entfernen und erhalten wieder einen Simplizialkomplex. Dieser Vorgang wird als Kollaps bezeichnet und ist ein Beispiel für eine Homotopieäquivalenz von Simplizialkomplexen. Insbesondere sind die Homologiegruppen des kleineren Komplexes isomorph zu den Homologiegruppen von $\mathcal{K}$ und beide Simplizialkomplexe haben die gleiche Anzahl von Löchern. Wie ein Kollaps aussieht und wieso er die Anzahl der Löcher unverändert lässt, können wir gut am Beispiel in Abbildung 6 erkennen.

In unserem Komplex der nicht-chromatischen Skalen können wir sukzessive alle acht- und siebentönigen Skalen mithilfe von Kollapsen entfernen. Wenn wir zum Beispiel aus der ersten achttönigen Skala in Abbildung 4 den Ton $C$ entfernen, dann erhalten wir eine siebentönige Skala, die man nur auf eine Weise zu einer achttönigen nichtchromatischen Skala erweitern kann. Also können wir mit diesen beiden Skalen einen Kollaps durchführen. Als weiteres Beispiel betrachten wir die C-Dur-Skala. Hier können wir den Ton $D$ entfernen, sodass die resultierende sechstönige Skala in nur einer maximalen nicht-chromatischen Skala enthalten ist. Demnach können wir die C-Dur-Skala und ihre sechstönige Teilskala kollabieren. Auf diese Art können wir alle sieben- und achttönigen Skalen aus $\mathcal{K}_{N C}$ entfernen. Dies bedeutet, dass die drei 5-Sphären in $\mathcal{K}_{N C}$ (die aus sechstönigen Skalen bestehen) außen herum höherdimensionale (nämlich sieben- und achttönige) Skalen geklebt haben, so wie das ausgefüllte Dreieck an das nicht-ausgefüllte Dreieck in Abbildung 6 geklebt ist. Übrigens ist es nicht möglich, die sechstönigen Facetten von $\mathcal{K}_{N C}$ zu kollabieren.

Es bleibt die Frage, wie unsere drei Sphären genau aussehen. Insbesondere wollen wir verstehen, aus welchen sechstönigen Skalen die einzelnen Sphären bestehen. Dazu betrachten wir den übermäßigen Dreiklang $C-E-G \sharp$. Wenn wir diesen aus unseren 12 Tönen entfernen, erhalten wir die in Abbildung 7 dargestellte neuntönige Skala, die der französische Komponist Messiaen verwendet hat.

Diese Skala enthält keine nicht-chromatischen Skalen mit sieben oder acht Tönen, aber 27 nicht-chromatische Skalen mit sechs Tönen. Diese erhält man wie folgt: Aus den 


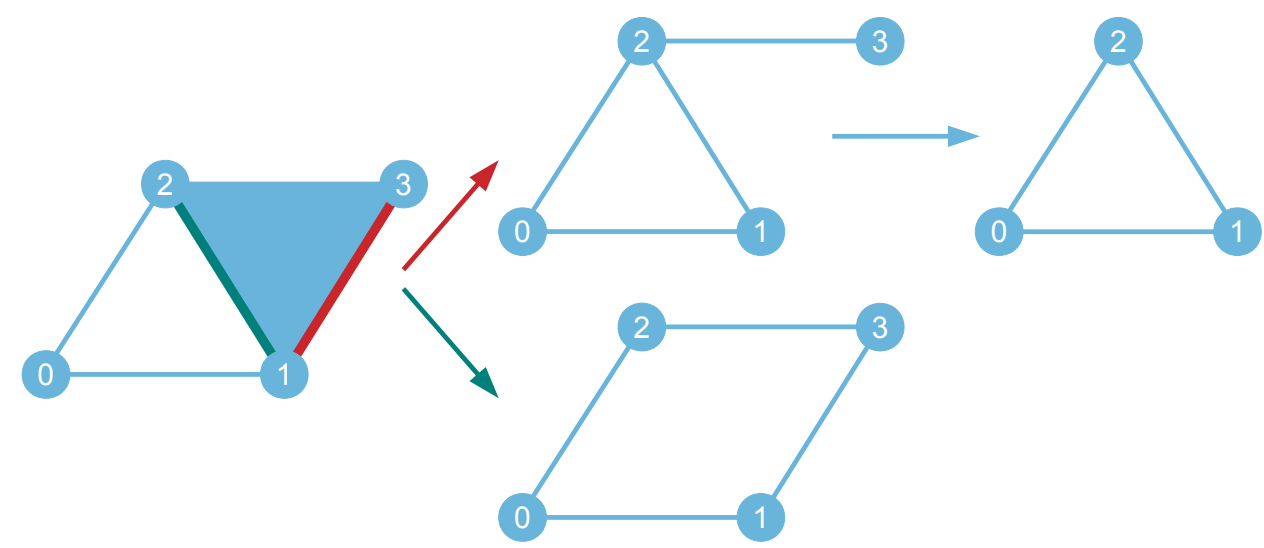

Abbildung 6. Der linke Simplizialkomplex hat 3 Facetten, aber nur eine davon kann kollabiert werden: $\{1,2,3\}$. Dabei kann man entweder die innere Kante (grün) oder eine der beiden äußeren Kanten (z. B. rot) entfernen. Im ersten Fall kann man danach keinen weiteren Kollaps durchführen. Im zweiten Fall ist genau ein weiterer Kollaps möglich.

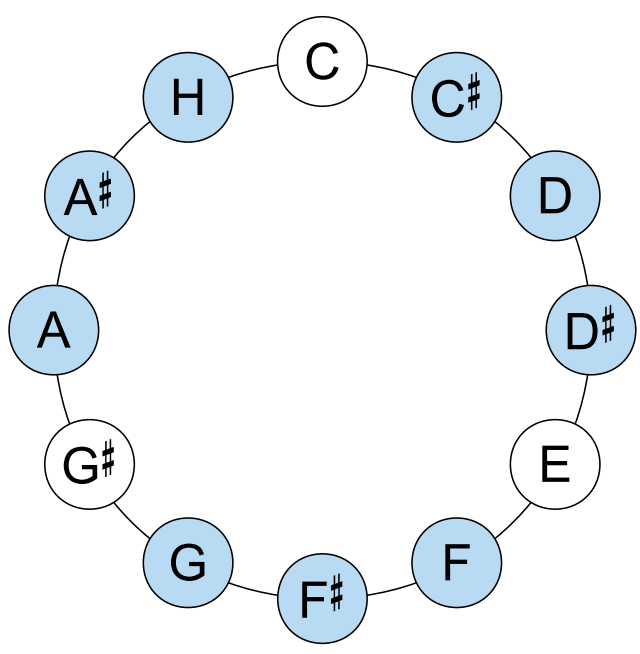

Abbildung 7. Messiaens neuntönige Skala

drei Tönen $C \sharp-D-D \sharp$ wählen wir genau zwei Töne (dafür gibt es drei Möglichkeiten), dann aus den Tönen $F-F \sharp-G$ weitere zwei Töne (wieder drei Möglichkeiten) und zum Schluss wählen wir zwei Töne aus $A-A \sharp-H$ (ebenfalls drei Möglichkeiten). Unsere polymake-Analyse ergibt, dass diese 27 sechstönigen Skalen und deren Teilskalen eine unserer drei 5-Sphären bilden.

Wie bei den verminderten Skalen in Abbildung 4 (hier waren es drei) gibt es insgesamt vier verschiedene neuntönige Messiaen-Skalen. Jede dieser vier Skalen enthält je 27 nicht-chromatische sechstönige Skalen, welche eine Sphäre in $\mathcal{K}_{N C}$ bilden. Nun haben wir aber behauptet, dass es nur drei Löcher in $\mathcal{K}_{N C}$ gibt. Das liegt daran, dass diese vier Messiaen-Sphären in $\mathcal{K}_{N C}$ nicht unabhängig voneinander sind. Dieses Prinzip können wir am Skelett eines Tetraeders gut erklären: Immer wenn wir den Simplizialkomplex in Abbildung 8 zeichnen, so sehen wir nur drei 1-dimensionale Löcher, obwohl es vier 1-Sphären (nicht-ausgefüllte Dreiecke) gibt.
Wir können nachrechnen, dass je drei der vier MessiaenSphären tatsächlich unabhängig voneinander in $\mathcal{K}_{N C}$ sind, d.h. dass diese eine Basis des Vektorraums $H_{5}\left(\mathcal{K}_{N C}\right)$ bilden. Zuletzt bemerken wir noch, dass jede der beschriebenen 5-Sphären genau drei der sechstönigen Facetten von $\mathcal{K}_{N C}$ enthält. Dies kann man zum Beispiel in Abbildung 7 erkennen. Die gezeigte Skala enthält genau eine der zwei Ganztonskalen und genau zwei der vier übermäßigen Skalen. Weiterhin schneiden sich je zwei verschiedene Messiaen-Skalen genau in einer sechstönigen Facette von $\mathcal{K}_{N C}$. Drei verschiedene Messiaen-Skalen schneiden sich in einem übermäßigen Dreiklang, d.h. in einem ausgefüllten Dreieck wie in Abbildung 5. Insbesondere können wir auf dem Schnitt von je zwei Messiaen-Sphären eine Reihe von Kollapsen durchführen, sodass wir am Ende den gemeinsamen übermäßigen Dreiklang erhalten, und wir können diesen Dreiklang solange kollabieren, bis nur noch ein Punkt übrig ist. Daher kann man aus topologischer Sicht sagen, dass die drei Sphären an einem Punkt verklebt sind. 


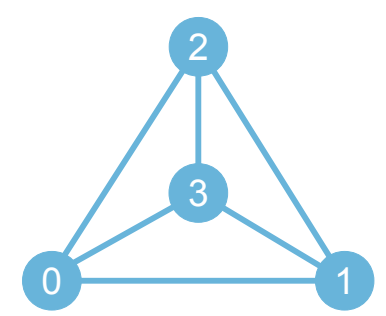

Abbildung 8. Simplizialkomplex mit den Facetten $\{0,1\},\{0,2\},\{0,3\}$, $\{1,2\},\{1,3\},\{2,3\}$

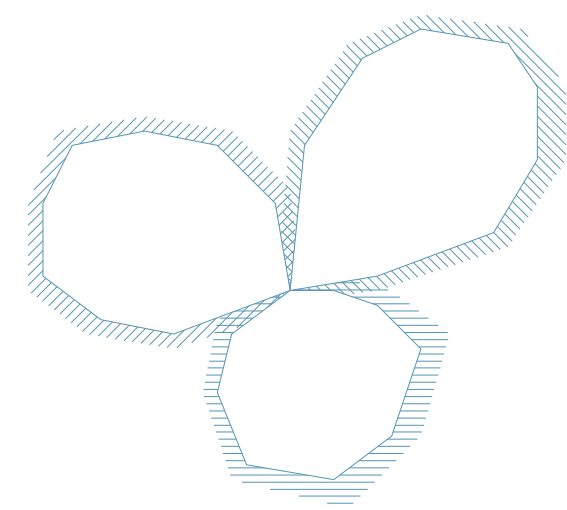

Abbildung 9. Schematische Darstellung der Topologie von $\mathcal{K}_{N C}$
Wir können uns also $\mathcal{K}_{N C}$ so vorstellen, dass man drei 6dimensionale Luftballons an einem Punkt zusammenhält und außen herum einen höherdimensionalen Bezug aufklebt.

Nun haben wir sowohl die 57 Facetten des Simplizialkomplexes $\mathcal{K}_{N C}$ als auch dessen topologische Struktur aus der Sicht der Mathematik und aus der Perspektive der Musik betrachtet. In den Mitteilungen der DMV 22 (4) ist bereits ein Artikel über „Mathematik und Musik?“ des Mathematikers und Pianisten Christian Krattenthaler erschienen, der die Grundthese vertritt, dass Mathematik und Musik eigentlich nichts miteinander zu tun haben. Wir sind jedoch zu dem Schluss gekommen, dass dies nicht immer der Fall ist, da der Simplizialkomplex $\mathcal{K}_{N C}$ sowohl mathematische als auch musikalische Bedeutung hat und noch viele weitere Zusammenhänge zwischen musikalischer Praxis (z. B. Skalenwechsel) und Mathematik untersucht werden können.

\section{Literatur}

[1] Ernst Ulrich Deuker. On the Way to a Grammar of Free Musical Speech: A Pentatonic Approach to Improvisation. Books on Demand, 2016.

[2] Ewgenij Gawrilow und Michael Joswig. polymake: A framework for analyzing convex polytopes. In Polytopes - Combinatorics and computation (Oberwolfach, 1997), volume 29 of DMV Sem., pages 43-73. Birkhäuser, Basel, 2000.

[3] Dimitry Kozlov. Combinatorial Algebraic Topology, volume 21 of Algorithms and Computation in Mathematics. Springer-Verlag Berlin Heidelberg, 2008.

[4] Heiner Zieschang Ralph Stöcker. Algebraische Topologie: Eine Einführung. Mathematische Leitfäden. Vieweg+Teubner Verlag, 1994.

[5] Walt Weiskopf Ramon Ricker. The Augmented Scale in Jazz: A Player's Guide. Jamey Aebersold, 2010.

[6] Frank Sikora. Neue Jazz-Harmonielehre: Von der Theorie zur Improvisation. Schott Music GmbH, 2012.

[7] Walt Weiskopf. Understanding the Diminished Scale: A Guide for the Modern Jazz Player. Jamey Aebersold, 2015.

Kathlén Kohn, Institut für Mathematik, MA 6-2, Technische Universität Berlin, Straße des 17. Juni 136, 10623 Berlin, kohn@math.tu-berlin.de

Ernst Ulrich Deuker, Goethestraße 75, 19053 Schwerin

Kathlén Kohn studierte Mathematik und Informatik an der Universität Paderborn. Seit dem Abschluss beider Master 2015 ist sie Doktorandin in der algebraischen Geometrie an der Technischen Universität Berlin. Zudem wirkt sie in diversen Musikprojekten (z. B. bei der Band Sh'lonk) und auf Veranstaltungen als Gastmusikerin an Cello und Klavier mit.

Ernst Ulrich Deuker studierte Mathematik an der FU Berlin. Drei Tage nach Abschluss des Studiums brach er zur ersten Tour seiner damaligen Band "Ideal" auf (1981). Später engagierte er sich mehr im nicht-kommerziellen Kulturbetrieb, drehte mit der Künstlerin Gabo gemeinsam experimentelle Super-8-Filme und veranstaltete Performances (u. a. „Das Optische Konzert“). Er entdeckte das seltene Instrument Kontrabassklarinette für sich und setzt es heute in verschiedenen Avantgarde-Projekten ein (z. B. bei „Affäre Bela B.“, Musik im Spannungsfeld zwischen den Kompositionen Béla Bartóks und freier Improvisation). Er ist auch als Musikpädagoge tätig und veranstaltet auf der Basis seines Improvisationslehrbuchs Workshops im In- und Ausland. 